\title{
Adopción contingente de tecnología de producción en el sector cerámico español. Un estudio empírico.
}

\author{
J.ALBORS, J.L.HERVAS, P.MARQUEZ \\ Depto.Org.Empresas, UPV
}

\begin{abstract}
Resumen. La literatura de estrategia y gestión ha considerado los factores situacionales como la base de la teoría de contingencia en la gestión de empresas. Las estrategias de tecnología de producción siguen esta pauta y tienden a adaptarse al contexto competitivo de la organización. Este trabajo tratará de demostrar esta hipótesis en el caso de la industria cerámica española.

Este sector se concentra en un cluster muy competitivo en la Comunidad Valenciana. Sin embargo, la perspectiva de la globalización ha traído a escena nuevos competidores en el ámbito mundial, como por ejemplo del Este asiático. Este hecho, el origen artesanal de la industria, un entorno cambiante con necesidad de un mayor enfoque al cliente y las propias características del proceso industrial, han propiciado la alineación de las estrategias de producción de tecnología más con la demanda de factores situacionales que con los objetivos de las organizaciones.

Considerando este escenario, los autores han llevado a cabo dos encuestas en una muestra representativa del sector. Estas contemplan las respuestas de las empresas a preguntas relacionadas con la gestión, el diseño y marketing, así como a las estrategias de tecnología de producción.

El análisis estadístico de los resultados de las encuestas concluye con una clasificación de las empresas según sus estrategias y resultados económicos, en grupos claramente definidos. De igual modo, el estudio concluye que la supervivencia de las empresas dependerá de la tendencia de las organizaciones para adaptar estrategias flexibles y proactivas (en términos de tecnología y marketing) alineadas con su entorno competitivo.
\end{abstract}

Palabras clave: Gestión de producción, contingencia, clusters.

Contingent adoption of production technology in the spanish tile ceramic cluster. An empirical study.

Strategy and management literature has considered situational factors as a basis for a contingency theory of firm management. Firm production technology strategies follow this trend and tend to adapt to the firm competitive context. This paper will try to demonstrate this hypothesis in the case of the tile ceramic industry in Spain. This industry concentrates in a very competitive cluster in the East of Spain. However the globalisation perspective has brought new global competitors from East Asia. This fact, a changing customer environment and the industry process characteristics, shape technology production strategies with demand situational factors rather than with firm objectives.

Considering this scenario, two surveys were carried out by the authors covering a representative sample of the industry. These contemplated the firm's reply to questions related to management, design and marketing as well as technology production strategies.

The statistical analysis of the survey results concluded in a classification of the industry firms according to their strategies as well as to their financial economic results in defined groups. The study concluded also that firm survival would depend on the firm tendency to adapt flexible and proactive strategies (technology and marketing wise) aligned with their competitive environment.

Keywords: production management, contingent, clusters

\section{INTRODUCCIÓN. ESTADO DEL ARTE. OBJETIVOS}

La influencia de los factores contingentes en la estructura de las organizaciones, así como la adopción de tecnología por las mismas ha sido tratada por diversos autores. El estudio desarrollado por Burns y Stalker (1) se centró en las relaciones de la organización con su entorno. Lawrence y Lorsch (2) propusieron la teoría de contingencia para explicar como diversos entornos demandan diferentes requisitos de las organizaciones. Woodward (3) contribuyó a esta discusion con una taxonomía de las modalidades de tecnología de producción.

Con relación a los modelos de producción, Toni y Tonchia (4) han revisado la literatura académica relacionada con la manufactura flexible y propuesto una taxonomía para clasificar ésta. Otros autores han analizado los factores contingentes que influencian la adopción de tecnologías de producción $(5,6$, 7).

La globalización, las nuevas modalidades de marketing, o los cambios en el contexto socioeconómico han inducido nuevas formas de organización de la producción. Estos nuevos paradigmas difieren de las aproximaciones clásicas Tayloristas o Fordistas (8) y han recibido atención de la literatura académica y práctica, especialmente en las aplicaciones relacionadas con la industria de montaje y la producción en masa. Estos modelos se han denominado Manufactura de 
Clase Mundial (9), Producción Ajustada (10)ํㅜ, Producción enfocada a la innovación (11), Producción estratégica flexible (12), Método Toyota (13), etc. Sin embargo, se han propuesto interpretaciones contingentes de la evolución de esos modelos. Algunas consideran especificidades relacionadas con los países o los sectores industriales. Otras, siguen aproximaciones estratégicas siguiendo la línea de Skinner (14) considerando, además, las competencias y el contexto competitivo de la empresa (15). Esta línea de investigación defende la idea de la estrategia de producción como una competencia competitiva (ver 17 y 18$)^{2}$.

Spina (19) ha analizado la controversia relacionada con la adopción de modelos de producción por parte de las organizaciones por motivos estratégicos o contingentes. Este autor apunta tres consideraciones con relación al rol de las contingencias: (a) Prácticas innovadoras en los sistemas de producción tales como JIT, Kanban, QFD, MRPII y sus adaptaciones contingentes requeridas por las transferencia de un país a otro; (b) modelos de producción basados en contingencias externas y decisiones estratégicas de las organizaciones; y (c), paradigmas de producción que incluyen modelos y técnicas. Ello requiere la adaptación al sector, al país y a la organización. Esta escuela de pensamiento (12) estableció el término Producción Estratégica Flexible SFP (por sus siglas en inglés) asumiendo estrategias con enfoques múltiples y flexibles, que incluyen la integración horizontal de los procesos de negocio en la organización y la implicación de los recursos humanos.

Es en esta línea de pensamiento en la que se desarrollará este artículo. El objetivo del presente trabajo es doble. En primer lugar, el estudio analiza los factores estratégicos de gestión y comercialización de la empresa con respecto a su tecnología de producción, al objeto de evidenciar, empíricamente, qué factores organizativos explican el posicionamiento tecnológico de producción en las empresas cerámicas estudiadas. En segundo lugar, el trabajo explora la adopción de las diferentes estrategias tecnológicas de producción con el desempeño empresarial

Debe subrayarse que ambos objetivos suponen una aportacion relevante y novedosa al estado del arte sobre el tema, como se analizará posteriormente. Este trabajo se estructura del siguiente modo. Primero, se describirá la industria y el contexto del mercado global de la misma: El cluster cerámico azulejero español. Segundo, se discutirá la metodología, la selección de la muestra y se presentarán los resultados del análisis estadístico. Finalmente, se terminará con las conclusiones y propuestas futuras de investigación.

\section{EL CLUSTER CERÁMICO AZULEJERO ESPAÑOL}

Tradicionalmente la industria cerámica azulejera ha estado dominada por unos pocos países que concentraban la mayor parte de la producción cerámica. Esta ha resultado influida por la disponibilidad de materias primas y tecnologías de fabricación. Bajo este contexto, se reconocen dos industrias azulejeras, especialmente la italiana y la española, como líderes a nivel mundial. Elo ha sido facilitado por las diversas discontinuidades tecnológicas que ha experimentado la industria cerámica $(20,21)$.

Este sector está influido tecnológicamente por los suministradores, de acuerdo con la taxonomía de Pavitt (22). El liderazgo del cluster azulejero español es reciente, precisamente debido a la absorción, por parte de los productores españoles, de las innovaciones generadas por la industria de bienes de equipo italiana. Ello fue, a su vez, seguido por los esfuerzos (coronados por el éxito) de la industria española de esmaltes para desarrollar productos innovadores que mejoraron radicalmente los procesos de fabricación (como por ejemplo, la Monococción).

\subsection{Estructura y perfil de las empresas españolas de producción azulejera}

Una de las principales características del sector es la alta concentración de la industria en la provincia de Castellón, ubicada en el Este de España. Aproximadamente el 94\% de la producción española total se origina en esta área geográfica que concentra el $76 \%$ del total de empresas productoras, constituido por 226 empresas.

Sólo el $20 \%$ de las empresas cuenta con más de 250 trabajadores. La mayoría de los productores españoles (54.8\%) tiene menos de 50 empleados y sólo 7 de ellos más de 500 . Por esta razón el tamaño promedio de las empresas oscila entre 50 y 100 empleados, lo que significa que son en su mayoría pequeñas y medianas empresas (PYMES). El mismo perfil presenta la facturación, donde sólo 3 empresas facturan más de 90 millones de Euros y 22 facturan entre 30 y 90 millones de euros (21).

$\mathrm{Al}$ ser una industria influida por las innovaciones de los proveedores, serán las empresas más grandes (o medianas líderes) las que usualmente lideren la implantación de normas y procedimientos, así como la incorporación de nuevas tecnologías. Estas firmas son más independientes de los fabricantes de maquinaria para la incorporación de tecnología, sistemas, etc. Las firmas más pequeñas siguen los movimientos

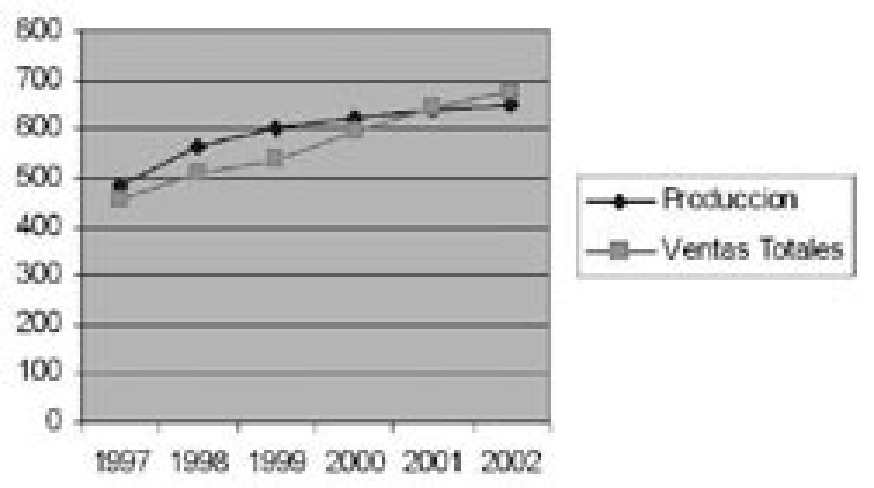

Fig. 1- Evolución de producción española y sus ventas totales (ASCER 2004). Miles de metros cuadrados y Millones de Euros.

de las líderes.

Por otra parte, la productividad ha aumentado un $62 \%$ desde 1990. La figura 1 muestra el crecimiento acumulado en los últimos años, confirmando dicha evolución, debido principalmente a las mejoras en términos de tecnología y productividad. La fabricación española representa el $45 \%$ de la producción total de la Unión Europea y el 10,5\% del total mundial.

Sin embargo, ambas industrias: italiana y española vienen sufriendo recientemente la fuerte competencia de las economías del Este afrontando nuevos retos (23). Dichas economías se han beneficiado de niveles bajos de salarios y disponibilidad de tecnología de los fabricantes de maquinaria 
italianos y productores de esmaltes españoles. Esta situación está teniendo una influencia decisiva en el cambio del enfoque competitivo de las empresas españolas desde una aproximación basada en la reducción de costes a otra basada en el valor agregado y donde la diferenciación, el diseño, los servicios de distribución y el enfoque al cliente aumentan su rol competitivo $(21,24)$.

\subsection{Planificación de la producción en las empresas Españolas}

Mientras el prensado, cocción, clasificación y empacado son procesos que se encuentran bien automatizados y la maquinaria necesaria se suministra en paquetes completos, el esmaltado y la decoración son, aún, procesos influenciados por técnicas aún artesanales y se encuentran lejos de los procedimientos estandarizados y en los que las habilidades y conocimientos de los operarios son fundamentales. Es precisamente en esta parte del proceso donde radican los retos de la planificación de producción. Las dificultades están asociadas con la repetición de las órdenes de fabricación manteniendo las características del producto, como por ejemplo, patrones de tonos. Así, hasta ahora la mayoría de los fabricantes han optado por la fabricación de grandes lotes de productos que se mantienen en stock (25). Dos estudios recientemente publicados nos permiten introducir al lector en la discusión sobre la aproximación estratégica de los sistemas de operaciones en las empresas cerámicas azulejeras españolas. El primero (26) relaciona las prioridades en la gestion de la producción manifestadas por una muestra de empresas con los objetivos estratégicos definidos por las empresas. El segundo (27) analiza los sistemas de programación de la producción utilizados en el sector cerámico. En esta dirección, para las empresas españolas, el aspecto más crítico en relación con la producción es el cumplimiento de los plazos de entrega. Esto explica el hecho que 50\% de las empresas intenta producir contra pedidos, pero sin el uso de ningún modelo estadístico de predicción del volumen de producción. Excepto en algunos casos, la herramienta más usual es una simple hoja Excel basada en estadísticas anteriores. Más aún, la optimización de la producción y la utilización de maquinaria, así como la reducción de costos de inventario y fabricación son aspectos con poca relevancia para la mayoría de las empresas en la planificación de la producción.

El tipo de software utilizado por las empresas se limita, en la mayoría de los casos, al uso de hojas de cálculo (100\%) $\mathrm{y}$ bases de datos $(80 \%)$. Estas herramientas son comunes a todas las firmas encuestadas en el estudio al que hemos hecho referencia. No obstante, en algunas empresas grandes y medianas el uso de sistemas para la planificación de recursos de la empresa (ERP, por sus siglas en inglés) y software a medida, ha llegado a ser herramientas relevantes que aunque ayudan en la tarea de planear la gestión, no son, por sí solas, suficientes para optimizar la planificación de la producción. De esta forma, es muy frecuente que el gerente de producción se vea forzado a planificar la producción manualmente y tomando en cuenta, el gran número de productos, formatos y líneas, los resultados pueden ser muy limitados.

Así, y aunque la producción cerámica azulejera se encuentra altamente automatizada, la mayoría de las empresas, no utilizan métodos para optimizar la producción, de acuerdo con los principales objetivos de la organización. Ello permitiría resolver adecuadamente las dificultades que se presentan en la actualidad y al mismo tiempo responder a los requerimientos del mercado en términos de diversificación y diferenciación de productos.

Los citados autores encuentran también grupos estratégicos, siempre definidos por los objetivos manifestados, asociados a la adopción de los sistemas de gestión de las operaciones (27).

\section{METODOLOGÍA}

\subsection{Marco analítico. Aproximación Contingente}

La utilización de las tecnologías de información por parte de las empresas ha sido reconocida como medio para reducción de costos (28), el desarrollo de valor añadido (29), la medición del éxito del negocio (30), la creación de ventaja competitiva (31) o el desarrollo de estrategias de gestión del conocimiento (32) entre otros.

Sin embargo, la adopción de Sistemas de Tecnologías de Información, especialmente en las PYMES, no es un hecho planificado sino que tiende a ser un proceso reactivo y contingente $(33,34)$. Este depende también de la fase de desarrollo en la que se encuentra la empresa (35), así, la planificación de Sistemas de Información es más frecuente en las PYMES que se encuentran en su etapa de madurez $(36 ; 37)$, ello en función de su tamaño.

Levy et al. (34) analizan la adopción de sistemas de información como función de la estrategia de la firma enfocada hacia la reducción de costos o hacia la agregación de valor. Otros factores contingentes son el enfoque al cliente, el entorno competitivo, el enfoque hacia la innovación, etc. Estos autores proponen un modelo llamado Modelo del Enfoque Dominante para la adopción de Sistemas de Información por parte de las organizaciones, que constituye la base de nuestra metodología. El modelo clasifica la adopción de sistemas de información (SI) en dos dimensiones del enfoque estratégico: Reducción de costes y dominancia del cliente (que preferimos cambiar por el término enfoque al cliente, modificando el modelo en este sentido) y valor agregado. Para este caso aplicaremos el modelo modificado para el análisis de adopción de tecnologías de gestión y control de la producción.

Este modelo combina esta aproximación con la clasificación de tecnologías de información de Earl (37) en tres categorías: Apoyo a la gestión, relaciones con el cliente y producción. La figura 2 describe el modelo esquemáticamente. Dependiendo del enfoque estratégico de la firma y de la intensidad de su enfoque al cliente, se pueden clasificar en cuatro tipos diferentes de tecnología. Eficiencia es el caso que se presenta cuando la empresa adopta una estrategia de reducción de costes y un nivel bajo de enfoque al cliente. Este enfoque se basa, es en este caso, en el control financiero y un uso de la

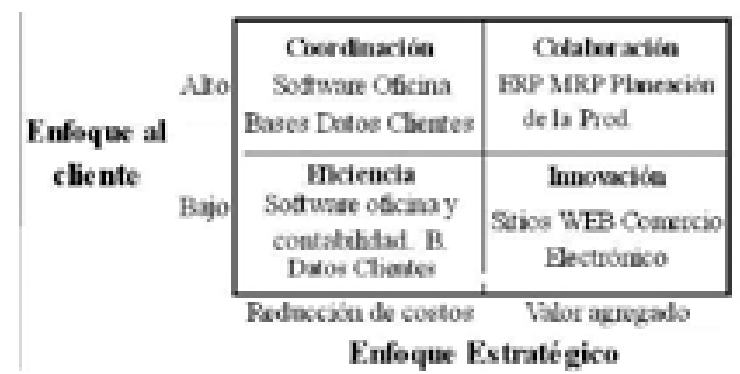

Fig. 2- Modelo del enfoque dominante de SI (Adaptado de Levy et al, 2001 y modificado por los autores). 
tecnología de tipo reactivo. Las herramientas que utilizaran las empresas en este caso serán simples, tales como software de oficina o programas de contabilidad.

Colaboración se presenta cuando la empresa adopta una estrategia de valor agregado y un alto grado de enfoque al cliente. Así, la empresa adoptará herramientas de gestión de producción más sofisticadas como ERP, MRP o planificación de la producción. Si la empresa adopta una estrategia de reducción de costes pero está altamente enfocada al cliente, nos referiremos al caso de Coordinación, donde las herramientas utilizadas serán software de oficina, programas de contabilidad y bases de datos de clientes. Finalmente, la Innovación se presenta cuando la organización adopta una estrategia de valor agregado y tiene un bajo nivel de enfoque al cliente, y las herramientas estarán conformadas por software de oficina, programas de contabilidad, páginas web o comercio electrónico.

Las hipótesis que trataremos de probar son las siguientes:

H1: La adopción de tecnologías relacionadas con los sistemas de producción estarán relacionadas con el tamaño de la empresa como una función de su fase de madurez.

H2: Las empresas estarán localizadas en la matriz de enfoque estratégico de acuerdo con su enfoque estratégico y/o enfoque al cliente según un modelo de distribución normal.

\subsection{Selección de la Muestra}

Este estudio forma parte de un proyecto, que nuestro grupo de investigación ha estado desarrollando en los últimos tres años con el objetivo de analizar la industria azulejera europea. El estudio fue financiado por un proyecto europeo (MONOTONE), con el objetivo de optimizar la gestión de fase de decoración.

El mismo se ha basado en el análisis de los datos económicos existentes, así como en la bibliografía del sector, la visita a las ferias sectoriales más importantes en Europa y Estados Unidos y la realización de 48 entrevistas con gerentes de empresas del sector cerámico azulejero español. El tamaño de la muestra es representativo del sector español (nivel de error $\leq 6,53 \%$ ) de acuerdo con el tamaño de las empresas.. La figura 3 muestra la composición de la muestra de acuerdo con el tamaño de las empresas (38).

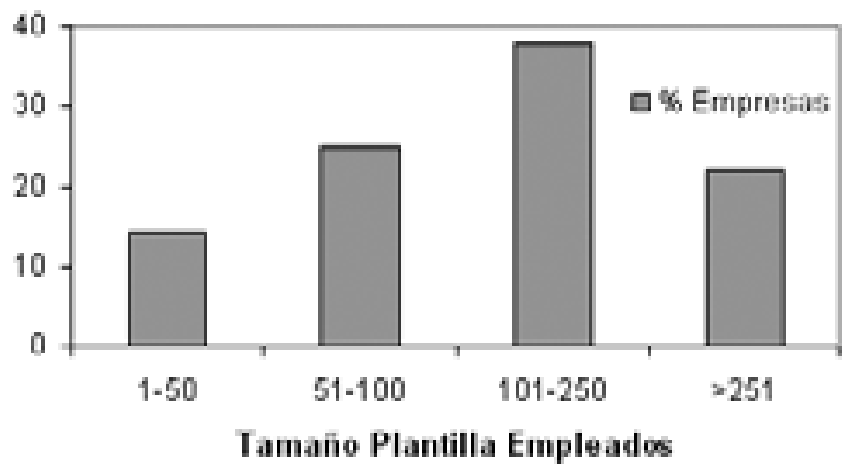

Fig. 3- Composición de la muestra de acuerdo con el tamaño de las empresas encuestadas.

El cuestionario comprende varios aspectos de la gestión de la empresa tales como su tamaño, aproximación estratégica, enfoque al diseño, gestión de conocimiento, gestión de imagen, y algunas preguntas específicas relacionadas con la gestión de la producción. Las variables, que han sido construidas para este análisis, se describen en la tabla 1.

TABLE I. VARIABLES UTILIZADAS EN LA ENCUESTA

\section{Grupo I: Variables de Gestión General y Variables Estratégicas.}

Design $($ Enfoque en diseño) = número de colaboraciones en diseño + existencia de diseño $+\%$ de personal de diseño de la firma + conocimiento de tendencias de clientes.

Maktgstrategy (Estrategia de Marketing) = esfuerzo en publicidad + portafolio segmentado y diferenciado + acuerdos de distribución $+\%$ de facturación en exportaciones + No. Oficinas de marketing $+\%$ personal de marketing empleada por la firma + contratos de marketing + exhibiciones internacionales atendidas + existencia de propios canales de distribución.

Knowledgemanagement (Gestión de Conocimiento) = $\%$ de graduados universitarios + horas de capacitación anuales.

Firm size $($ Tamaño de empresa $)=$ No. De empleados + producción en $\mathrm{m} 2$ + facturación.

ColabR\&D centres (Colaboración con centros de $\mathrm{I}+\mathrm{D})=$ Colaboración RTD + numero de contratos.

ISO $=$ certificado como $14000+9000$

$\mathrm{ERP}=$ Firma con ERP

\section{Grupo II: Variables de gestión de producción}

ProdSched: si la producción es planificada contra stock (1) o los pedidos de los clientes (5).

Prodtechinnovativeness. Innovación enfocada hacia la maquinaria (1) o la gestión de la produccion (5).

Controlcorrect $=$ errores en las variables de producción controlados y corregidos.

ProdDatarecord. $=$ Variables de producción controladas y datos registrados.

Dataustilstartup $=$ los datos de las variables de producción se utilizan para nuevas puestas en marcha. Qualevel $=$ Nivel de calidad del producto final.

Quallevcontimpr. $=$ Nivel de calidad del producto final mejorado anualmente.

Formatchangespeed $=$ Velocidad con la cual se cambian los formatos y los productos.

Prodoproced $=$ Procedimientos de producción a nivel de gestión.

Barlackproced $=$ Barreras a la gestión de la producción debido a falta de procedimientos.

Barexcessvar $=$ Barreras debido al exceso de variables de proceso.

Conservad = Barreras a la gestión de la producción debido al conservadurismo del personal.

Techequip = Barreras a la gestión de la producción debido a control técnico de la maquinaria.

Lackprocknow $=$ Barreras a la gestión de la producción debido a la falta de conocimientos de los procesos. Compdrive $=$ Esfuerzo competitivo en costos (1), en diferenciación (5).

Innovfocus = Enfoque de innovación en costo (1), en calidad del producto (5).

Formrelsuppl $=$ Relaciones formales con proveedores bajas (1) a altas (5). 
TABLA IB. VALORES DE LAS VARIABLES UTILIZADAS EN LA ENCUESTA

\begin{tabular}{|c|c|c|c|c|c|}
\hline & \multicolumn{5}{|c|}{ Estadisticos descrip tivos $N=48$} \\
\hline & Variable & M inimo & Máximo & Media & Desv. tip. \\
\hline 1 & D iseño & 0 & 30 & 5,952 & 6,477 \\
\hline 2 & Tamaño & 41,7 & 1122,27 & 250,395 & 249,915 \\
\hline 3 & Colabcentroim as d & 0 & 6 & 2,146 & 1,833 \\
\hline 4 & EstrategiaM K T G & 4,68 & 27,2 & 14,610 & 5,402 \\
\hline 5 & GestConoc & 1 & 5,07 & 3,361 & 0,955 \\
\hline 6 & Iso & 0 & 2 & 0,771 & 0,857 \\
\hline 7 & ERP & 0 & 2 & 1,104 & 0,973 \\
\hline 8 & ProdSched & 1 & 5 & 3,396 & 1,086 \\
\hline 9 & Prodtechinnovativeness\# & 1 & 5 & 3,042 & 1,184 \\
\hline 10 & Controlcorrect & 1 & 5 & 2,938 & 1,327 \\
\hline 11 & ProdDatarecord & 1 & 5 & 2,813 & 1,468 \\
\hline 12 & Dataustilstartup & 1 & 5 & 3,250 & 1,407 \\
\hline 13 & Qualevel & 1 & 5 & 3,458 & 1,148 \\
\hline 14 & Quallevcontimpr & 1 & 5 & 3,313 & 1,371 \\
\hline 15 & Formatchangespeed & 1 & 5 & 3,250 & 1,280 \\
\hline 16 & Prodproced & 2 & 5 & 3,542 & 0,944 \\
\hline 17 & Barlackproced & 1 & 5 & 3,354 & 1,229 \\
\hline 18 & Barexcessvar & 1 & 5 & 3,438 & 1,109 \\
\hline 19 & Conservad & 2 & 5 & 3,479 & 0,945 \\
\hline 20 & Lackstand & 1 & 5 & 3,333 & 1,434 \\
\hline 21 & Formalrelsup 1 & 1 & 5 & 3,250 & 1,280 \\
\hline 23 & Innovfocus & 1 & 4 & 2,866 & 1,040 \\
\hline 24 & Techequip & 1 & 5 & 2,958 & 1,031 \\
\hline 25 & Lackprocknow & 1 & 5 & 3,313 & 1,257 \\
\hline 26 & Compdrive & 1 & 4 & 2,966 & 1,096 \\
\hline
\end{tabular}

Con el objetivo de representar una aproximación estratégica de la firma (enfoque de valor añadido), se ha construido una nueva variable como resultado de la combinación de las siguientes variables:

(Valor añadido) Valadded $=$ ColabR\&D + Maktgstrategy + Knowledgemagement + Controlcorrect+ ProdDatarecord + Qualevel + Dataustilstartup+ Formrelsuppl.

El test de fiabilidad para esta variable compuesta fue positivo con Alfa de Cronbach $=0,826$ y $\mathrm{p}<0,0001$

Igualmente, la variable que constituye el grado de enfoque al cliente por parte de la firma, representada por la siguiente ecuación:

(Enfoque al cliente) Customer focus $=$ Designfocus $+\mathrm{ISO}+$ vision + orgcom + medios ave + prodsched + prodtechinnovt + format change speed + prodprovced + ERP

El test de fiabilidad para esta variable compuesta fue positivo con Alfa de Cronbach $=0,740$ con $\mathrm{p}<0,0001$.

\section{ANÁLISIS ESTADÍSTICO DE LOS RESULTADOS}

Se ha desarrollado un Análisis Factorial para examinar que variables iniciales pueden explicar la varianza de la muestra. Este método se utiliza, tanto en la reducción de datos para identificar un número de factores mínimo que explique la varianza observada en un número mayor de variables, para generar hipótesis relacionadas con los mecanismos causales o para inspeccionar las variables en análisis subsiguientes (en este caso, el análisis cluster). El primer ejercicio concluyó con los siguientes resultados.

Tras diversos ejercicios y simulaciones, se redujeron las variables a tres componentes, cuya composición muestra la tabla 2. Los tres componentes que se muestran en la siguiente tabla, pueden explicar el $82,50 \%$ de la varianza de la muestra. Como se puede observar, desde un punto de vista contingente, las actividades de diseño resultan muy relevantes, así como aquellas asociadas con las actividades de I+D, la gestión de conocimiento de la empresa y las variables de gestión de producción. Todas ellas apunta a una organización con estructura relativamente formal, e indican el nivel de formalidad (rutinas) de las relaciones con los proveedores. El tamaño de la empresa parece tener un peso relevante. Como puede observarse, estas tres componentes utilizan 18 de las 26 variables iniciales utilizadas en la encuesta. Las variables no utilizadas no aportan información adicional relevante al análisis y podrían considerarse redundantes.

El componente 1 está asociado con las siguientes variables contingentes: estrategia basada en el enfoque al diseño, al nivel de calidad y su mejoramiento continuo, enfoque hacia innovaciones de tecnología de producción, tamaño de la empresa y su relación formal con los suministradores tecnológicos. Con relación a las variables de gestión de la producción, están asociadas con la planificación de la producción, procedimientos para corregir y recopilar datos relativos a las variables de proceso, así como con la utilización de estos para nuevos puestas en marcha y con la velocidad de cambio de los formatos.

TABLA II. ANÁLISIS DE FACTORES. MATRIZ DE COMPONENTES ROTADOS

\begin{tabular}{|l|l|l|l|}
\hline & \multicolumn{3}{|c|}{ Components } \\
\hline & 1 & 2 & 3 \\
\hline CollabRTDcenter & & & 0,693 \\
\hline Design activities & 0,599 & & \\
\hline Knowledge management & & & 0,759 \\
\hline ERP & & & 0,590 \\
\hline ProdSched & 0,923 & & \\
\hline Controlcorrect & 0,670 & & \\
\hline ProdDatarecord & 0,658 & & \\
\hline Dataustilstartup & 0,861 & & \\
\hline Qualevel & 0,897 & & \\
\hline Quallevcontiimpr & 0,888 & & \\
\hline Formatchangespeed & 0,898 & & \\
\hline Prodoproced & & 0,926 & \\
\hline Barlackproced & & 0,919 & \\
\hline Barexcessvar & & 0,923 & \\
\hline Conservad & & 0,946 & \\
\hline Firm size & 0,597 & & \\
\hline Prodtechinnovativeness $\#$ & 0,577 & & \\
\hline Formalrelsuppl & 0,892 & & \\
\hline
\end{tabular}

\begin{tabular}{|c|l|l|}
\hline $\begin{array}{l}\text { Kaiser-Meyer-Olkin. } \\
\text { Sample Measurement } \\
\text { Adequacy }\end{array}$ & 0,850 & \\
\hline Bartlett Sfericity Test & $\begin{array}{l}\text { Chi-square } \\
\text { Approx. }\end{array}$ & 1437,536 \\
\hline & Fl & 153 \\
\hline & Sig. &, 000 \\
\hline
\end{tabular}

Notas:

Método de extracción: Análisis de componentes principales.

Método de rotación: Normalización Varimax con Kaiser. La rotación ha convergido en 5 iteraciones.

El componente 2 está asociado básicamente con las barreras encontradas para establecer estándares y procedimientos de producción.

El componente 3 está relacionado con dos variables 
contingentes: aquellas relacionadas con la gestión del conocimiento e I+D y una variable de gestión de producción: la implantación de paquetes de software ERP en la empresa.

Posteriormente, se realizó un análisis de cluster con las tres componentes $(\mathrm{C} 1, \mathrm{C} 2$ y C3) como variables separadoras (Ver tabla 3).

Se obtuvieron cuatro clusters. El cluster número 2 representa un grupo de empresas con ciertos niveles de excelencia en la gestión, de acuerdo con sus procedimientos de producción. Este grupo exhibe los coeficientes más altos de estrategia de marketing y procedimientos de diseño, gestión de conocimiento y colaboración en actividades de I+D.

Más aún, si representamos los valores para las variables compuestas - enfoque al cliente y valor añadido - en un diagrama de dispersión, siguiendo los patrones del modelo de enfoque dominante, obtenemos la matriz que se presenta en la figura 3. Esta figura muestra igualmente el cluster de pertenencia en cada caso. presentaban un desempeño económico superior que el resto de las firmas de la muestra ${ }^{3}$.

\section{CONCLUSIONES}

El modelo de enfoque dominante propuesto por Levy et al (34), con las modificaciones realizadas por los autores, ha resultado ser muy útil para analizar la relación contingente entre la adopción de tecnologías y prácticas ligadas a los sistemas de gestión de la producción y el enfoque estratégico y táctico de la empresa, así como su etapa de madurez medida por su tamaño y el nivel de formalidad de sus procedimientos de gestión.

Las alternativas consideradas por este enfoque estratégico se pueden resumir en la reducción de costes o el enfoque de valor añadido, en la cadena de valor, por parte de la empresa. Otros factores contingentes que han demostrado tener influencia en este proceso de decisión son el enfoque al

TABLA III. CLASIFICACIÓN EN CLUSTERS DE ACUERDO CON LOS TRES COMPONENTES

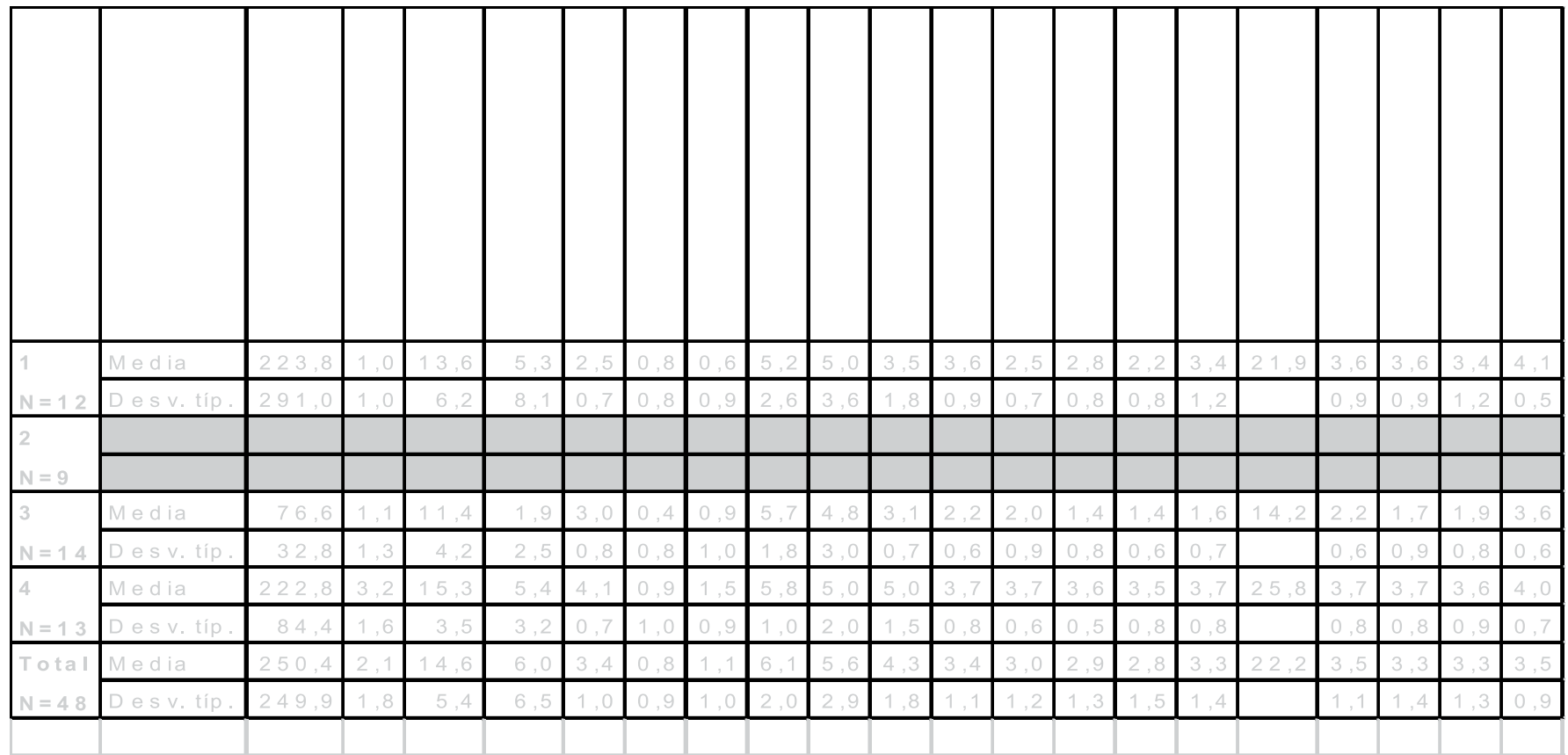

La muestra se distribuye con un patrón de dispersión longitudinal siguiendo la diagonal de la matriz y, en general, las empresas presentan valores bajos y medios para la variable de enfoque al cliente. Con pocas excepciones, los clusters obtenidos coinciden con los cuadrantes de la matriz. De esta forma, el cluster 2 coincide con el grupo de Colaboración (Cuadrante NE); el cluster 3 coincide con el grupo de Coordinación (Cuadrante SW); el cluster 4 lo hace con el grupo de Innovación (Cuadrante SE). Finalmente, el cluster 1 está distribuido entre los grupos de Coordinación e Innovación.

Estos clusters coinciden, en general, con aquellos desarrollados durante el desarrollo de las fases preliminares de nuestra investigación, cuando el enfoque estratégico era el objetivo de análisis (ver figura 4). Así, se habían identificado cuatro clusters de acuerdo con las siguientes variables: gestión de la imagen de la firma, portafolio de productos (existencia de diferenciación), colaboración con las instituciones externas de I+D (ITC, Alicer) y tamaño de la empresa (que se convierte en una variable de control). Un análisis más profundo demostró que las empresas pertenecientes al cluster de excelencia cliente, el contexto de nivel de competencia en el enfoque de la innovación, etc.

Los resultados han demostrado que la distribución de las empresas de la muestra sigue un patrón de dispersión diagonal en la matriz del modelo propuesto. Las empresas se concentran en tres cuadrantes correspondientes a los niveles más altos de valor añadido y los niveles más bajos de enfoque al cliente. Esos resultados confirman las conclusiones de las encuestas previas desarrolladas en las primeras fases de la investigación. El cluster cerámico español muestra un enfoque hacia el cliente débil y ciertas deficiencias en la gobernanza de las fases finales de la cadena de valor (Distribución, Servicios Post-venta, Colocación, etc. $)^{4}$. Esta situación aparece como contingente con el enfoque de gestión de producción de las empresas, así como con la utilización de sistemas de tecnologías de información en este aspecto.

Puede concluirse entonces que la primera hipótesis postulada es correcta. La adopción de tecnologías ligadas a los sistemas de gestión de la producción es contingente con el 


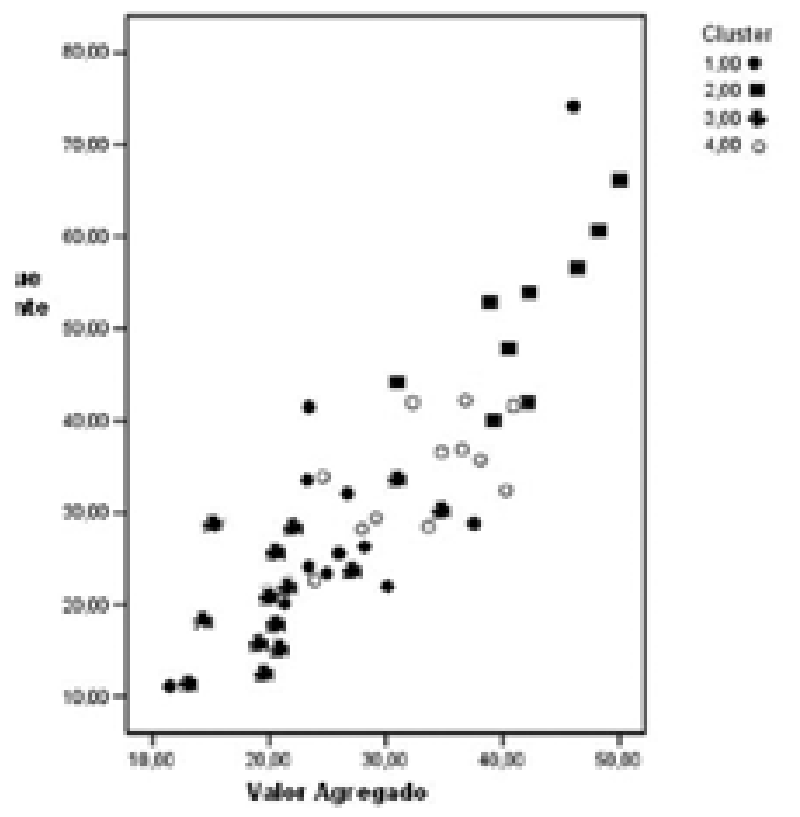

Fig. 4- Matriz del Modelo de enfoque dominante construida para la muestra analizada.

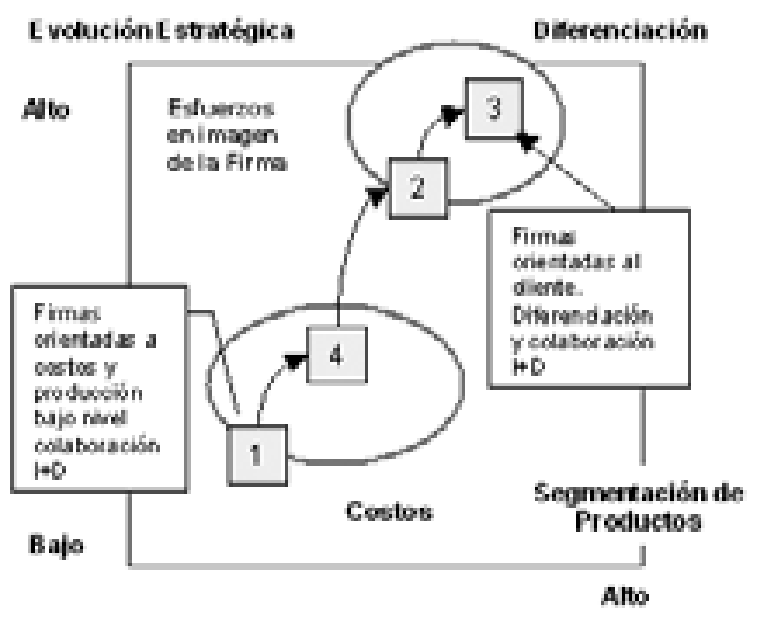

Fig. 5- Identificación de clusters estratégicos.

tamaño de la empresa como función de su etapa de madurez. Esto constituye una aportación relevante al estado del arte ya que considera la evolución contingente de la empresa. Con relación a la segunda hipótesis postulada, se ha demostrado que esta es parcialmente correcta. Las empresas se distribuyen en un modelo modificado de enfoque estratégico dominante. Ello, de acuerdo con su enfoque estratégico y/ o su enfoque hacia el cliente, en un modelo de distribución que no es normal debido a la existencia de estrategias parciales en las empresas, sin mostrar un enfoque definido hacia el cliente final. El aporte de este trabajo consiste en identificar las estrategias de la empresa, no únicamente por sus objetivos definidos en una encuesta como apuntaban investigaciones anteriores, sino por las actividades tácticas desplegadas por la empresa. Además, se ha encontrado una correlación entre el desempeño económico y el enfoque de sus sistemas y tecnologías de gestión de la producción. Ello conduce a una aplicación práctica directa del trabajo presentado.
Como trabajo de futuro se apunta la recopilación de información adicional en el cluster azulejero italiano para verificar las hipótesis de trabajo. Los datos macroeconómicos relacionados con este cluster muestran un enfoque al cliente más fuerte en productos pertenecientes a segmentos de precio más altos, relaciones más fuertes con los canales de distribución y políticas de imagen más elevadas hacia los cliente. Un estudio comparativo con esta metodología reforzaría la validación de nuestro modelo. Con relación al cluster azulejero español nuestra investigación se enfoca actualmente hacia la eficiencia de los diversos canales de distribución y su relación con los fabricantes.

\section{BIBLIOGRAFÍA}

1. T. Burns y G.M. Stalker, (1961), The management of innovation, Tavistock, Publ., London. Pp. 119-122.

2. P.R. Lawrence, J.W. Lorsch, (1967), Organization and Environment: Managing Differentiation and Integration. Boston, MA: Harvard University.

3. J. Woodward, (1958), Management and Technology, HMSO, London.

4. A. Toni, S. Tonchia, (1998) Manufacturing flexibility: a literature review, Int. Jour. Production Research, Vol. 36, 6, 1587-1617.

5. K.K. Boyer,P.T. Ward, K.G. Leong, (1996), Approaches to the factory of the future, an empirical taxonomy, Journal of Operations Management, 14, pp. 297-313.

6. R. Cagiliano, G. Spina, (2000), Advanced manufacturing technology and strategically flexible production, Journal of Operations Management, 18, pp. 169-190.

7. A. Das, J. Jayaram, (2003) "Relative Importance of Contingency Variables for Advanced Manufacturing Technology," Int. Jour. Production Research, Vol. 41, 18, 4429-4452.

8. M. Kenney, R. Florida, (1984), Japan's role in a post fordist age, Futures, April, pag. 136-151.

9. R.J. Schonberger, (1986), World class manufacturing: the lessons of simplicity applied, Free Press, New York.

10. J.P. Womack, D.T. Jones, (1990), The machine that changed the world, Mc Millan Press, London.

11. M. Kenney, R. Florida, (1993), Beyond mass production: the Japanese system and its transfer to the U.S., Oxford University Press, New York.

12. G. Spina, E. Bartezzaghi, R. Cagliano, D. Dranizier, H. Boer, A. Bert, (1996), Strategically flexible production: the multifocused manufacturing paradigm, Int. Journal Operations and Production management, vol.3, nº, pags.4-13.

13. J. Liker, (2004), The Toyota Way, McGraw-Hill, N.Y.

14. W. Skinner, (1969), Manufacturing: the missing link in corporate strategy, Harvard Business Review, May June, pp. 136-145.

15. R.H. Hayes, G.P. Pisano, (1994), Beyond world class the new manufacturing strategy, Harvard Business review, Jan.-Febr., pp. 77-86.

16. Kochan, T.A., Lansbury, R. D, MacDuffie, J.P., (1997), After Lean Production: Evolving Employment Practices in the World Auto Industry, ILR Pres, Ithaca, N.Y. 12

17. R.H. Hayes, G.P. Pisano, (1996), Manufacturing strategy. At the intersection of two paradigms shifts, Production and Operations Management, special issue on manufacturing strategy, vol. 5, nº1, Spring pp. 5-42.

18. Clark, K.B., (1996), Competing through manufacturing and the manufacturing paradigm, Production and Operations Management, special issue on manufacturing strategy, vol. 5, $\mathrm{n}^{\circ} 1$, Spring pp. 42-57.

19. Spina, G., (1998), Manufacturing paradigms versus strategic approaches: a misleading contrast, Int. Journal of Operation and production management, 18,8 , pg. 684-690.

20. J. Albors, (2002), Networking and technology transfer in the Spanish Ceramic Tiles Cluster. Its role in the sector competitiveness, The Journal of Technology Transfer, Vol. 27(3), pp. 263-273.

21. J. Albors, J.L Hervás (2006), La industria cerámica europea en el siglo XXI. Retos tecnológicos y desafíos de la próxima década, Bol. Soc. Esp. Ceram. Vidrio, V., 45 [1] 13-21. (2006)

22. K. Pavitt, (1984), Sectoral patterns of technical change: towards to taxonomy and to theory", Research Policy n ${ }^{\circ} 13$

23. E. Criado, E. Sanchez, M Regueiro, (2004) La industria cerámica española, ¿ante un cambio de ciclo?, Bol. Soc. Esp. Ceram. V., 43 [1] 85-101, (2004).

24. E- Criado, M. Regueiro y E. Sánchez. “La industria cerámica en España (1990-2000)" Bol. Soc.Esp. Ceram. V., 40 [6] 413-428. (2001)

25. I. Tortajada, G. Peris-Fajarnés, M. Aguilar, P. Latorre. “Análisis del proceso de clasificación cerámico" Bol. Soc. Esp. Ceram. V., Vol. 45 [1] 22-27. (2006)

26. E. Vallada, C. Maroto, R. Ruiz, B. Segura, Análisis de la programación de la producción en el sector cerámico español, Bol. Soc. Esp. Ceram. V., 44 [1] 39-44. (2005) 
27. B. Segura, E. Vallada, C. Maroto, R. Ruiz, "Análisis del sistema de operaciones en empresas del sector cerámico español", Bol. Soc. Esp. Ceram. V., 43 [6] 929-932 (2004).

28. J. Ward, P. Griffiths, (1990), Strategic planning for IS, Wiley \& Sons, Chichester, U.K.

29. S. Zuboff, (1988) The age of the smart machine, Heineman Publishers, Oxford.

30. R.D. Galliers, (1991) Strategic IS planning, myths, reality and guidelines form successful implementations. European Journal of Information systems, vol. 1, pp.55-67.

31. M.E. Porter, V.E. Millar, (1985), How information gives you competitive advantage, Harvard Business Review, July August, pp. 141-160.

32. M.J. Earl, (1996) Knowledge strategies: propositions from two contrasting industries, in Information Management, Earl, M.J., (editor), Oxford University Press, pp. 36-53, N.Y.

33. B. Dankbaar, (1998), Technology management in technology-contingent SMEs, Int. Journal of Technology Management, Vol.15, 1/2, pp. 70-81.

34. M. Levy, P. Powell, P. Yetton, P., (2001), SMEs: aligning IS and the strategic context, Journal of Information Technology, 16, pp. 133-144.
35. R.G. McMahon, (1998), Stage models of SME Growth Reconsidered, School of Commerce Research paper series: 98-5, School of Commerce Flinders University, Melbourne.

36. G. Reid, (1999), Information Development in the small firm, Information and management, 35, 248-269.

37. M.J. Earl, (1989) Management strategies for Information technology, Prentice Hall, London.

38. N.C. Churchill, V.L. Lewis, (1983) The five stages in small business growth Harvard Business Review, 61(3), May-June, pp. 30-52.

39. J.L.Hervas J., Albors, J.I.Dalmau, (2004), Asociación de grupos estratégicos y performance empresarial. evidencia empírica a través de la relación con las estrategias genéricas., Proceedings ACEDE, Congress, Murcia.

Recibido: $\quad 10.10 .05$

Aceptado: 13.03 .06 